\title{
Distinction of Patterns within Time-Series Data Using Constellation Graphs
}

\author{
Mayumi Oyama-Higa ${ }^{1}$, Michihiko Setogawa $^{2}$, and Teijun $\mathrm{Miao}^{3}$ \\ ${ }^{1}$ Department of Integrated Psychological Science, Kwansei Gakuin University \\ 1-1-155, Ichibancho,Uegahara, Nishinomiya-City,662-8501, Japan \\ oyama@kwansei.ac.jp \\ ${ }^{2}$ Research \& Development Center, Hitachi Systems \& Services, Ltd. \\ 10-70, 2-Chome, Nanbanaka, Naniwa-ku, Osaka, 556-0011, Japan \\ m-setogawa@hitachi-system.co.jp \\ ${ }^{3}$ Chaos Technical Research Laboratory and CCI Incorporation \\ 3-1-2401, Ryodocho, Nishinomiya-City, 662-0841, Japan \\ t-miao@tokyo.cci-web.co.jp
}

\begin{abstract}
Constellation graphs for time-series data are very effective tool for displaying a physio-psychological index. In this study, we recorded fingertip pulse waves of elderly subjects, carried out chaos analysis on the plethysmogram data thus obtained, and examined their relationship with dementia. We discovered the Lyapunov exponent of the time series had a clear relationship with the severity of dementia and the communication skill of the elderly subjects. We could clearly demonstrate a relationship between dementia and the Lyapunov exponents using constellation graphs of the time series data.
\end{abstract}

\section{Introduction}

Measuring fingertip pulse waves, which are biomedical signals, is easier than electroencephalography because it is less restrictive and more convenient. In this study, we conducted experiments to examine whether senile and other types of dementia have any relationship with the fingertip pulse waves. The study subjects were residents of an elder care home and persons receiving day-care at the same facility. The fingertip pulse waves of each subject were measured and chaos analysis was carried out to obtain three minutes of time series data. The chaos attractor, entropy, and the largest Lyapunov exponent were calculated as time series data. In order to obtain the criteria for estimating the subjects' quality of life and severity of dementia, we gathered data on the activities of daily living (ADL) measured by the subjects' caregivers and on criteria of different levels of dementia severity. The relationship between the $\mathrm{ADL}$ indices and the results of the chaos analysis and the severity of dementia was analyzed. We discovered that the mean value and standard deviation of the largest Lyapunov exponent of the time series had a clear relationship with the severity of dementia and the communication skill of the elderly subjects. We also examined the relationship between dementia and communication skills and could clearly demon- 
strate a relationship between dementia and the largest Lyapunov exponents using constellation graphs of the time series data.

\section{Data analyzed}

Informed consent was obtained from both the subjects' care home and their families. There were 179 subjects (139 females and 40 males), who ranged in age from 65 to 100 years (mean 83.4). Measurements were made between August and November 2003. The temperature, systolic blood pressure, diastolic blood pressure, and pulse rate were measured before recording the plethysmograms.

\section{Measuring fingertip pulse waves and analyzing plethysmograms}

\subsection{Method of measurement}

The pulse waves were measured using a photoplethysmography sensor (CCI $\mathrm{BC} 2000$ ) using the following procedure. We gave the subjects at least 10 minutes each, to become accustomed to the surroundings in a room maintained at $25^{\circ} \mathrm{C}$. They were allowed to sit comfortably in a chair with both hands placed in a relaxed manner on a desk (at a height that was comfortable for writing). The subjects kept their eyes open while the measurements were made on the left index finger for 180 seconds. The signals were A/D converted. Digital data sampled at a frequency of $200 \mathrm{~Hz}$ and a resolution of 12 bits were fed to a PC.

\subsection{Method of chaos analysis}

For the time series data $x(i)$, with $i=1, \ldots, \mathrm{N}$ obtained from the fingertip pulse waves, the phase space was reconstructed using the method of delays. Assuming that we create a $d$-dimensional phase space using a constant delay $\tau$, the vectors in the space are generated as d-tuples from the time series and are given by:

$$
\mathbf{X}(i)=(x(i), \ldots, x(i-(d-1) \tau))=\left\{x_{k}(i)\right\}
$$

where $x_{k}(i)=x(i-(k-1) \tau)$, with $k=1, \ldots, d$. In order to correctly reconstruct the phase space, the parameters of delay, $\tau$, and embedding dimensions $d$ should be chosen optimally [4]. In time series recorded from human finger photoplethysmograms, we chose the parameters $\mathrm{T}=50 \mathrm{~ms}$ and $d=4$, as in references [1] and [2].

In the reconstructed phase space, one of the important measures of complexity is the largest Lyapunov exponent $\lambda_{1}$. Considering $\mathbf{X}(t)$ is the evolution with time of some initial trajectory $\mathbf{X}(0)$ in the phase space, it is given by 


$$
\lambda_{1}=\lim _{t \rightarrow \infty} \lim _{\varepsilon \rightarrow 0} \frac{1}{t} \ln \frac{\left|\delta \mathbf{X}_{\varepsilon}(t)\right|}{|\varepsilon|}
$$

where

$$
\begin{gathered}
\delta \mathbf{X}_{\varepsilon}(t)=\mathbf{X}(t)-\mathbf{X}_{\varepsilon}(t) \\
\varepsilon=\mathbf{X}(0)-\mathbf{X}_{\varepsilon}(0)
\end{gathered}
$$

for almost all initial difference vectors $\varepsilon=\mathbf{X}(0)-\mathbf{X}_{\varepsilon}(0)$. We estimated $\lambda_{1}$ using the algorithm of Sano and Sawada [3]. $\lambda_{1}$ describes the divergence and instability of the orbits in the phase space.

The largest Lyapunov exponents, $\lambda_{1}$, were calculated for a basic window of 8,000 points $(40 \mathrm{sec})$. The $180 \mathrm{sec}(36,000$ points $)$ were covered by sequentially sliding, 200 points $(1 \mathrm{sec})$ at a time and $\lambda_{1}$ was determined for each window.

\section{Activities of daily living (ADL) and dementia level data of the elderly subjects}

Data recorded by the persons caring for the subjects in the care home was used as the ADL data. The dementia data used were those recorded by healthcare professionals. For the ADL, each activity of daily living, such as walking, eating, toileting, bathing, dressing, and grooming was assigned to one of three care dependence categories: a) can do independently although it takes a long time; b) needs some assistance; and c) totally dependent on assistance. Each subject's communication skill was also assigned to one of the three levels: a) can communicate normally; b) can communicate to some extent; and c) can hardly communicate.

Data on dementia obtained from the healthcare professionals were categorized into the five grades of dementia: 0 : none, 1: mild, 2 : moderate, 3 : severe, and 4: very severe.

\section{Pulse wave parameters and analysis of ADL indices and severity of dementia}

We examined whether the values of the largest Lyapunov exponent (group means and standard deviations) had any significant correlation with each of the factors - sex, age, body temperature, systolic blood pressure, diastolic blood pressure, pulse rate, walking, eating, toileting, bathing, dressing, grooming, communication skill, and severity of dementia - in order to examine the relationship between the ADL indices and dementia with the pulse wave data.

We found that only communication skill and the severity of dementia were significantly correlated with the mean and standard deviation of the Lyapunov exponent. 
Figs. 1 and 2 show the relationship of these factors with mean and standard deviation of the Lyapunov exponent, respectively.

The Lyapunov exponent is a measure of the divergence of the attractor trajectory. It became clear from the above results that communication skill and dementia were related to the deviation of the Lyapunov exponent and that elderly persons who could not communicate well had less deviation of the exponent compared with those who could communicate well. We observed similar relationships with dementia.
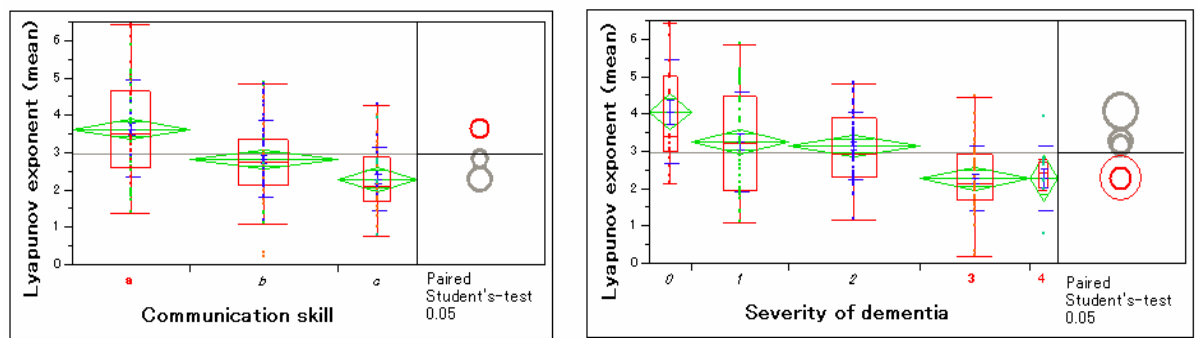

Fig. 1 Relationship of the mean Lyapunov exponent with communication skill and dementia
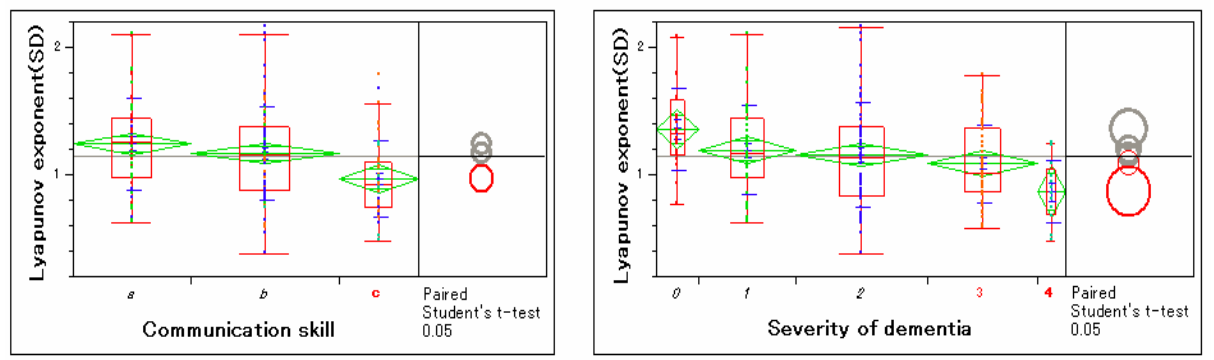

Fig. 2 Relationship between the standard deviation of the Lyapunov exponent with communication skill and dementia

\section{Representation of dementia in a constellation graph}

If we could represent the changes in the Lyapunov exponent obtained by sliding within the 180 -second time interval visually, it would be possible to measure the changes in the severity of dementia easily.

We realized such a visual representation by preparing constellation graphs[5]. In these constellation graphs, the numerical data of a time series were converted into angles between 0 and $180^{\circ}$, and the vectors of the same length were joined and depicted on a semicircular graph. The maximum and minimum values were set automatically from the values of the Lyapunov exponent. Each line represents the data for one subject. The smaller the Lyapunov exponent, the closer the vector is to the bottom right of the constellation graph. As the value of the Lyapunov exponent increases, 
the line moves to the left in the graph. The line is straighter when the standard deviation is small, and is bent more when it is large.

Figure 3 shows the relationship between dementia and the Lyapunov exponents obtained from the analysis. The five levels of dementia are shown in different colors. Subjects with more severe dementia have their lines more to the right side of the graph. The subjects in the five different dementia categories were sorted according to severity and five subjects around the median value were selected from each category to prepare this graph. Fig. 4 is similar to Fig. 3, but shows the relationship between communication skill and the Lyapunov exponent.

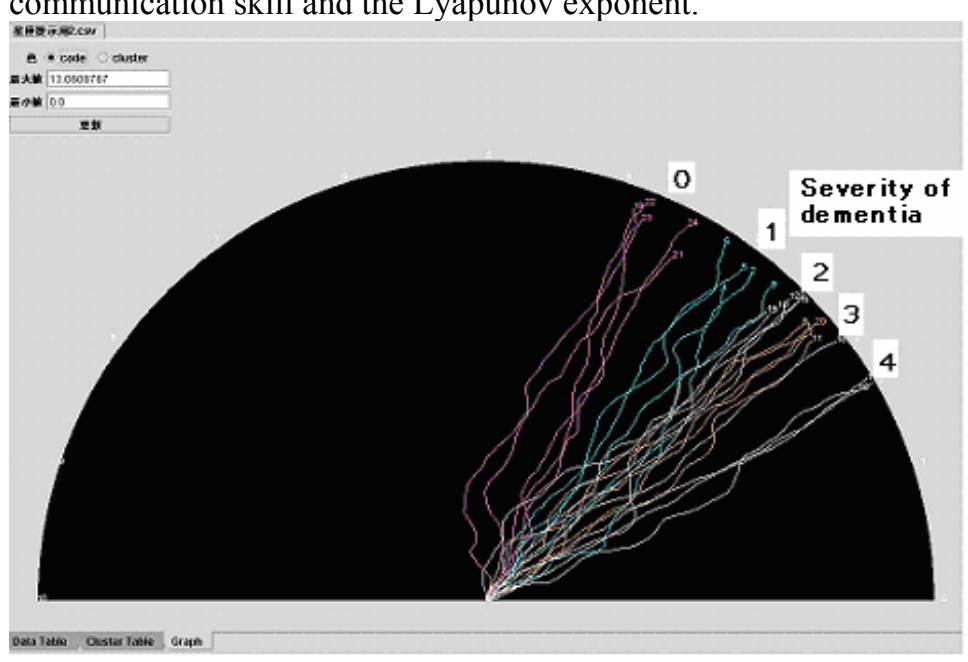

Fig. 3 The relationship between the severity of dementia and the Lyapunov exponents of the time series (each line represents one subject)

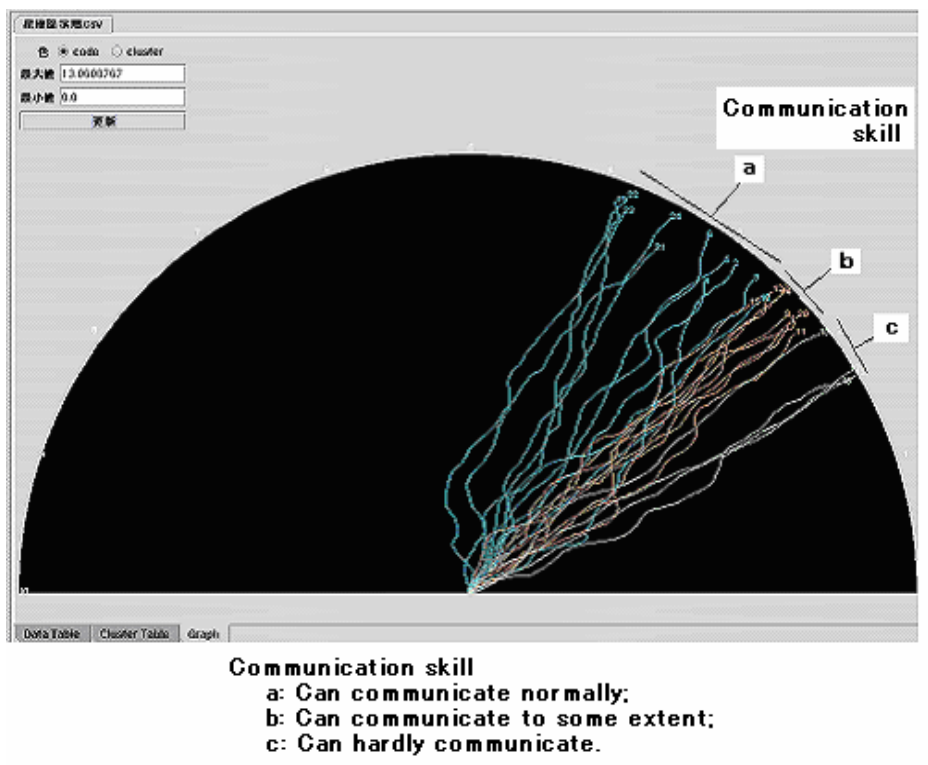


Fig. 4 The relationship between communication skill and the Lyapunov exponents of the time series

\section{Conclusion}

The traditional view of homeostasis has been that when certain factors disturb vital signs, such as the pulse rate, respiration rate, blood pressure, and temperature, that are directly related to the maintenance of life, and push them outside the normal range, corrective feedback is activated to stabilize them, and stable values of these signs indicate the proper functioning of the control mechanisms of the living body. However, even when a healthy subject is placed at bed rest, the heart beat interval fluctuates irregularly. Respiration rate, blood pressure, and body temperature act similarly. In fact, the fluctuations of the heart rate are less prominent in elderly persons and sick persons.

In this study, changes in vital signs caused by aging were measured through fingertip pulse waves, represented as constellation graphs, and verified. We demonstrated that the Lyapunov exponent was related to dementia in elderly persons. Our research showed that the decrease in the divergence of the Lyapunov exponent in subjects with advanced senile dementia was similar to that observed in persons with depressive psychosis. We plan to advance this study further by obtaining detailed data on the changes in divergence during the time when a newborn infant becomes a young child and in patients with mental diseases, like depressive psychosis.

Acknowledgments: We thank the staff of the elder care home, Associate Professor Hirohashi of Seisen University, and Dr. Junko Tsujino and the students of the Oyama Laboratory of Kwansai Gakuin University for their help and cooperation in collecting data and taking measurements from our elderly subjects.

\section{References}

1. Tsuda I, Tahara T, Iwanaga I : Chaotic pulsation in capillary vessels and its dependence on mental and physical conditions. Int J Bifurcation and Chaos 2: (1992)313-324.

2. Sumida T, Arimitu Y, Tahara T, Iwanaga $H$ :Mental conditions reflected by the chaos of pulsation in capillary vessels. Int J Bifurcation and Chaos 10: (2000) 2245-2255.

3. Sano M and Sawada Y : Measurement of the Lyapunov spectrum from a chaotic time series. Phys. Rev. Lett. 55: (1985)1082.

4. Abarbanel HDI, Brown R, Sidorowich JJ, Tsimring LS: The analysis of observed chaotic data in physical systems. Rev Mod Phys 1993, 65: 1331-1392.

5 . Tokihiko Niwa, Kenji Fujikawa, Yoshikazu,Tanaka, Mayumi Oyama: Visual Data Mining Using a Constellation Graph . ECML/PKDD-2001,Freiburg 